\title{
Evaluation of Nonstructural 1 Antigen Assays for the Diagnosis and Surveillance of Dengue in Singapore
}

\author{
Kwoon-Yong Pok, Yee-Ling Lai,, Joshua Sng, ${ }^{1}$ and Lee-Ching $\mathrm{Ng}^{1}$
}

\begin{abstract}
Early and accurate diagnosis of dengue is imperative for disease surveillance, which helps in the control of dengue in endemic countries. In this study, we evaluated the performance of three commercially available dengue nonstructural 1 (NS1) antigen assays (Bio-Rad Platelia ${ }^{\mathrm{TM}}$ Dengue NS1 Antigen ELISA, PanBio Dengue Early ELISA, and Bio-Rad Dengue NS1 Antigen Strip test) and compared them with reverse-transcription polymerase chain reaction (RT-PCR) and other commercially available serological assays for the diagnosis of dengue. The analysis showed RT-PCR to be the most sensitive and specific $(100 \%)$ diagnostic method during the first 3 days of fever. The overall sensitivity of dengue NS1 antigen assays within the same period was $81.7 \%$, indicating their potential role as a cost-effective and convenient alternative method to RT-PCR for the diagnosis of dengue fever in a primary healthcare setting. However, reduced sensitivity in detecting secondary dengue infections was one of the drawbacks of dengue NS1 antigen assays. Nonetheless, it remains a useful assay for the early detection of dengue and hence could play an important role in routine surveillance efforts to control dengue outbreaks in Singapore.
\end{abstract}

Key Words: Dengue—Diagnosis—NS1 antigen—Serology—Surveillance.

\section{Introduction}

D ENGUE IS A MOSQUITO-BORNE viral disease affecting approximately 50-100 million individuals annually in more than 100 countries (Gubler 2002). Seventy percent of the 2.5 billion people at risk of dengue reside in Southeast Asia and Western Pacific region. Dengue virus (DENV) is a flavivirus comprising four distinct yet antigenically related serotypes (DENV1-4) in DENV antigenic complex (Calisher et al. 1989). Infection with any of the four serotypes can result in an illness with a wide clinical spectrum (Halstead 1989) ranging from a mild flu-like febrile episode to death due to dengue hemorrhagic fever and dengue shock syndrome. There has been a gradual increase in the global incidence of dengue since the year 2000 (World Health Organization 2009) which is believed to be due to factors such as rapid urbanization, expanding human population and activities, and increased global travel and geographical expansion of the primary vector, Aedes aegypti. Therefore, dengue is believed to pose a mounting challenge to the tropical and subtropical regions throughout the world.
As there is currently no specific therapy or commercially available vaccine to prevent or treat the infection, early detection of disease transmission and outbreaks is critical for surveillance programs that are aimed at minimizing morbidity by controlling disease spread. At present, laboratory confirmation of dengue relies on demonstration of the presence of DENV by (a) isolation of DENV from patient serum, (b) detection of viral RNA by reverse transcriptionpolymerase chain reaction (RT-PCR), and (c) detection of dengue-specific antibodies (immunoglobulin $\mathrm{M}[\mathrm{IgM}] / \mathrm{IgG}$ ) by using enzyme-linked immunosorbent assay (ELISA) (Pan American Health Organization 1994, Guzman and Kouri 1996, Vorndam and Kuno 1997). Each method has its own advantages and disadvantages that define criteria for their selection in different settings.

Viral isolation is laborious and requires approximately 7-10 days to obtain results. It is thus not suitable as front-line assays for early diagnosis of dengue infection. In contrast, viral RNA detection by PCR technology allows early diagnosis of infections as results can be obtained within hours (Lai et al. 2007). It can also be used for rapid serotyping of the virus

\footnotetext{
${ }^{1}$ National Environment Agency, Environmental Health Institute, Singapore, Singapore.

${ }^{2}$ Singapore Polytechnic, School of Chemical and Life Sciences, Singapore, Singapore.
} 
(Lanciotti et al. 1992, Lai et al. 2007). However, PCR is an expensive option for routine diagnostic purposes as it requires trained personnel and specialized laboratory equipments and reagents. The usage of dengue-specific antibody detection assays, though cost-effective, is limited in the early phase of disease as antibodies become detectable only around the fifth day upon the onset of disease. The antibody assays ideally require appropriately timed paired serum samples for the confirmation of an acute dengue infection (Pan American Health Organization 1994, Guzman and Kouri 1996, Vorndam and Kuno 1997). Over the past decade, several studies (Young et al. 2000, Huang et al. 2001, Alcon et al. 2002, Koraka et al. 2003, Xu et al. 2006) have shown that detecting dengue nonstructural 1 (NS1) protein could be a promising and effective alternative for the early diagnosis of dengue.

Dengue NS1 is a highly conserved $46-\mathrm{kDa}$ nonstructural glycoprotein that both exists as an intracellular, membraneassociated and as an extracellular form secreted from DENVinfected mammalian cells (Winkler et al. 1989). Clinical observations have shown that dengue NS1 antigen can be detected in the circulation during DENV infection and it elicits a specific immune response (Monath and Heonz 1996). Later studies further demonstrated the presence of dengue NS1 antigen in acute-phase sera of infected individuals. In cultures, the levels of NS1 protein correlated with infectious titers of DENV (Young et al. 2000, Alcon et al. 2002).

Over the recent past, several dengue NS1 antigen assays are available commercially for the detection of dengue NS1 antigen in human sera. Evaluation of these assays (Dussart et al. 2006, Kumarasamy et al. 2007, Blacksell et al. 2008, Lapphra et al. 2008) has demonstrated high sensitivity and specificity for DENV detection, particularly during the acute phase of the disease. In this study, we evaluated the performance of three commercially available dengue NS1 antigen assays, in comparison with other widely used dengue diagnostic assays, to determine the usefulness of NS1 antigen assays in early diagnosis of dengue in a primary healthcare setting in Singapore. This study could provide additional information to help improve laboratory diagnosis and demonstrates the importance of comprehensive evaluation of commercial kits before any large-scale application of a test. This could be of interest to those involved in the study of management of vector-born diseases.

\section{Materials and Methods}

\section{Serum samples}

Two sets of sera (set A and set B) constituting 433 blood samples from 321 suspected dengue cases collected during 2005-2007 were used in this evaluation.

Set A consisted of acute and convalescent sera collected from 112 patients suspected of having dengue as part of a broad study on dengue fever (Low et al. 2006). Acute samples were obtained within the first $72 \mathrm{~h}$ from the onset of fever, and the convalescent samples from the same individuals were collected on third to eighth day after the onset of fever.

Set B consisted of 209 nonpaired serum samples obtained from individuals suspected of having dengue. All samples of set $B$ were collected from the onset (day 1) to 8 days after the onset of fever at primary healthcare clinics in Singapore and sent to the Environmental Health Institute (EHI), a national public health laboratory, for routine diagnosis. Samples were
Table 1. Summary of Test Sera Used for the Evaluation of Dengue Nonstructural 1 Assays

\begin{tabular}{lccc}
\hline & $\begin{array}{c}\text { Set A } \\
\text { (112 paired } \\
\text { samples) }\end{array}$ & $\begin{array}{c}\text { Set B } \\
\text { (209 single } \\
\text { collection) }\end{array}$ & $\begin{array}{c}\text { Total cohort } \\
\text { of patients }\end{array}$ \\
\hline Confirmed dengue (D) & 52 & 109 & 161 \\
Primary dengue (P) & 31 & 84 & 115 \\
Secondary dengue (S) & 21 & 25 & 46 \\
Nondengue (ND) & 60 & 100 & 160 \\
\hline
\end{tabular}

stored at $-80^{\circ} \mathrm{C}$ until analysis by RT-PCR and dengue NS1 and serology assays.

\section{Detection and serotyping of DENV}

Real-time RT-PCR for the detection and serotyping of DENV was performed on 433 suspected dengue samples as previously described (Lai et al. 2007).

\section{Serological analyses}

Dengue IgM and IgG antibodies in 433 suspected dengue samples were determined using commercially available dengue antibody detection assays: Dengue Capture IgM ELISA ("Capture IgM"), Dengue Capture IgG ELISA ("Capture $\mathrm{IgG}^{\prime \prime}$ ), and PanBio Dengue Duo Cassette kit ("Dengue Duo"), in accordance with the manufacturer's instructions (Inverness Medical Innovations, Queensland, Australia).

\section{Dengue NS1 antigen assays}

Presence of dengue NS1 antigen in 433 suspected dengue samples was determined using three commercially available kits: Platelia ${ }^{\mathrm{TM}}$ Dengue NS1 Antigen ELISA ("Platelia"), Dengue NS1 Antigen Strip ("NS1 Strip"; Bio-Rad Laboratories, Marnes La Coquette, France), and PanBio Dengue Early ELISA ("Dengue Early"; Inverness Medical Innovations) assays. All tests were performed according to the manufacturers' instructions.

\section{Characterization of infection status}

"Recife" method (Cordeiro et al. 2009) was used in this study to classify the dengue infection status of analyzed samples. Different infection statuses were assigned to 321 patients suspected of having dengue (Table 1) based on the following criteria:

(1) Confirmed dengue infection (D) was defined as the presence of viral RNA, dengue-specific IgM antibodies, or high titer (equivalent to hemagglutination inhibition titer of $>2560$ ) of dengue-specific IgG antibodies (based on Capture $\operatorname{IgG}$ ) in test sera.

(2) Nonacute infection (ND) was characterized by the absence of virus RNA, anti-dengue IgM, and/or high titer IgG antibodies in sera.

(3) Primary dengue infection $(\mathrm{P})$ was defined as the absence of high titer of dengue-specific IgG antibodies (based on Capture IgG) in the acute sera sample and presence of anti-dengue IgM antibodies or viral RNA, followed by presence of anti-dengue IgG in convalescent sera sample. 


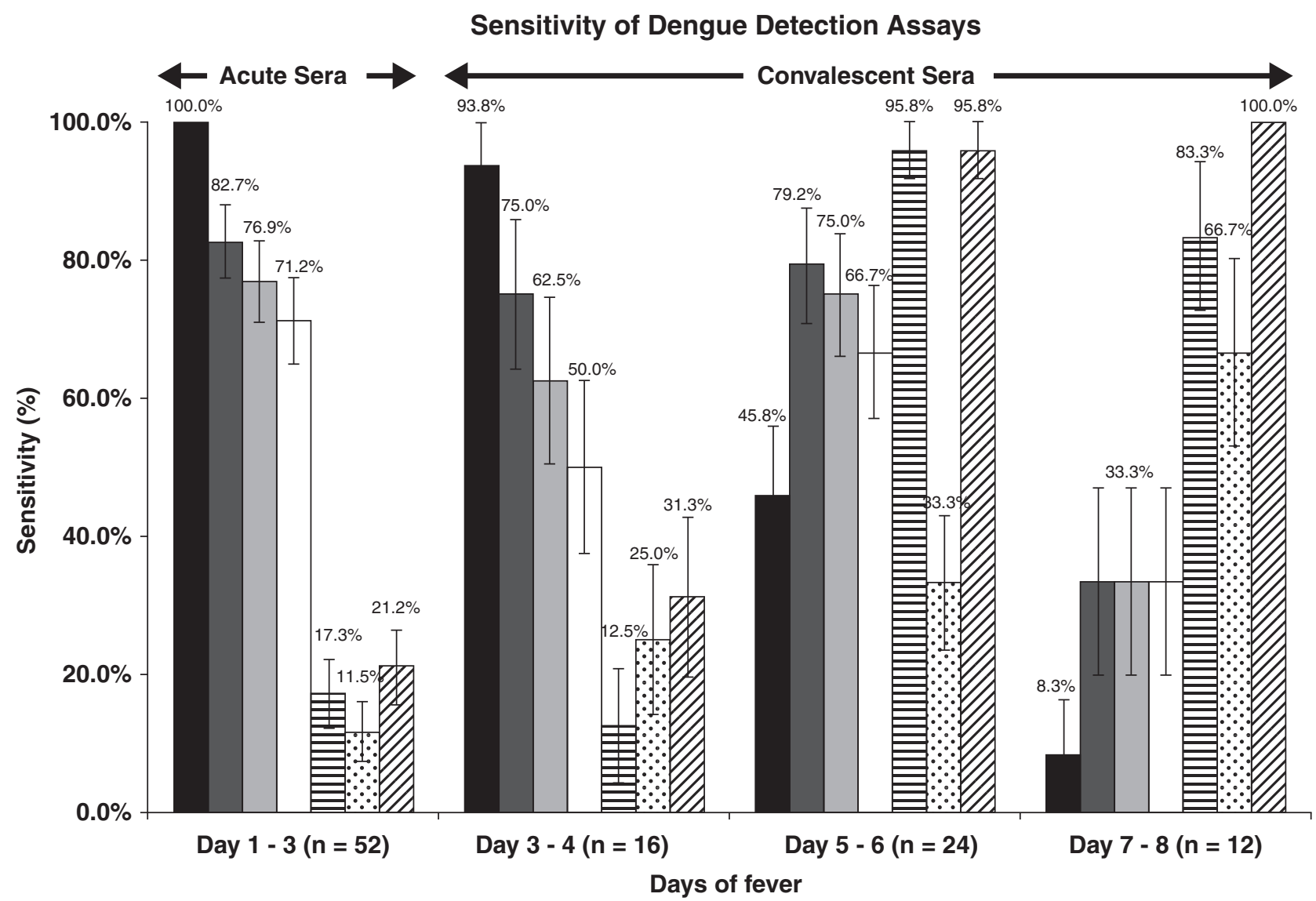

\begin{tabular}{|lll|}
\hline RT-PCR & $\square$ Bio-Rad Platelia Dengue NS1 Ag & $\square$ Dengue NS1 Ag STRIP \\
$\square$ Panbio Dengue Early & 目 Panbio Dengue Capture IgM & $\square$ Panbio Dengue Capture IgG \\
$\square$ Panbio Dengue Duo Cassette & & \\
\hline
\end{tabular}

FIG. 1. Longitudinal variation of the sensitivity of assays used for the diagnosis of dengue. The comparison was made using set A samples that consisted of paired serum of dengue-infected individuals collected within the first 3 days of fever (acute) and from days 3 to 8 (convalescent). The error bars show two standard deviations of the proportion of sensitivity for each bar.

(4) Secondary dengue infection (S) was defined as the presence of high titer (equivalent to hemagglutination inhibition titer of $>2560$ ) of dengue-specific IgG antibodies (based on Capture IgG) in the acute serum and/or viral RNA detection in the acute sera sample.

\section{Ethical considerations}

Written consent was obtained from each patient (or the patient's guardian) after explaining the research objectives. All data were handed confidentially and anonymously. This study was reviewed and approved by the Internal Review Board of the National Healthcare Group and National Environmental Agency, Singapore.

\section{Statistical analysis}

Diagnostic accuracy indices of sensitivity, specificity, positive predictive value (PPV), negative predictive value (NPV), chi-square, and Cohen's kappa values were calculated using SPSS for Windows software (version 13.0; SPSS, Chicago, IL). The sensitivity, specificity, PPV, and NPV for the assays were calculated as follows: sensitivity $=($ true positive $) /($ true positive + false negative); specificity $=($ true negative $) /($ true negative + false positive $) ; \mathrm{PPV}=($ true positive $) /($ true positive + false positive); NPV $=$ (true negative $) /($ false negative + true negative). The $95 \%$ confidence intervals (CIs) were calculated as estimates of the effectiveness of assays using $\pm 2 \times$ standard error of proportion (formula: $\sqrt{ }[p(1-p) / n]$ ). Diagnostic odds ratio (DOR) is the quotient between positive likelihood ratio $(\mathrm{LR}+)$ and the negative likelihood ratio (LR-). LR+ and LRwere calculated using the following formulas: [sensitivity/ $(1-$ specificity $)]$ and $[(1-$ sensitivity $) /$ specificity $]$, respectively.

\section{Results}

\section{Characterization of study sera based on Recife method}

Of 112 first collected sera of set A samples, 52 (46.4\%) were positive for dengue by RT-PCR and included all four serotypes: DENV1 $(n=19), \operatorname{DENV} 2(n=24), \operatorname{DENV} 3(n=8)$, and DENV4 $(n=1)$. The serological analysis of convalescent sera showed the presence of dengue IgM and/or high titer of IgG (based on Capture IgG) in all 52 RT-PCR-positive samples. Of 


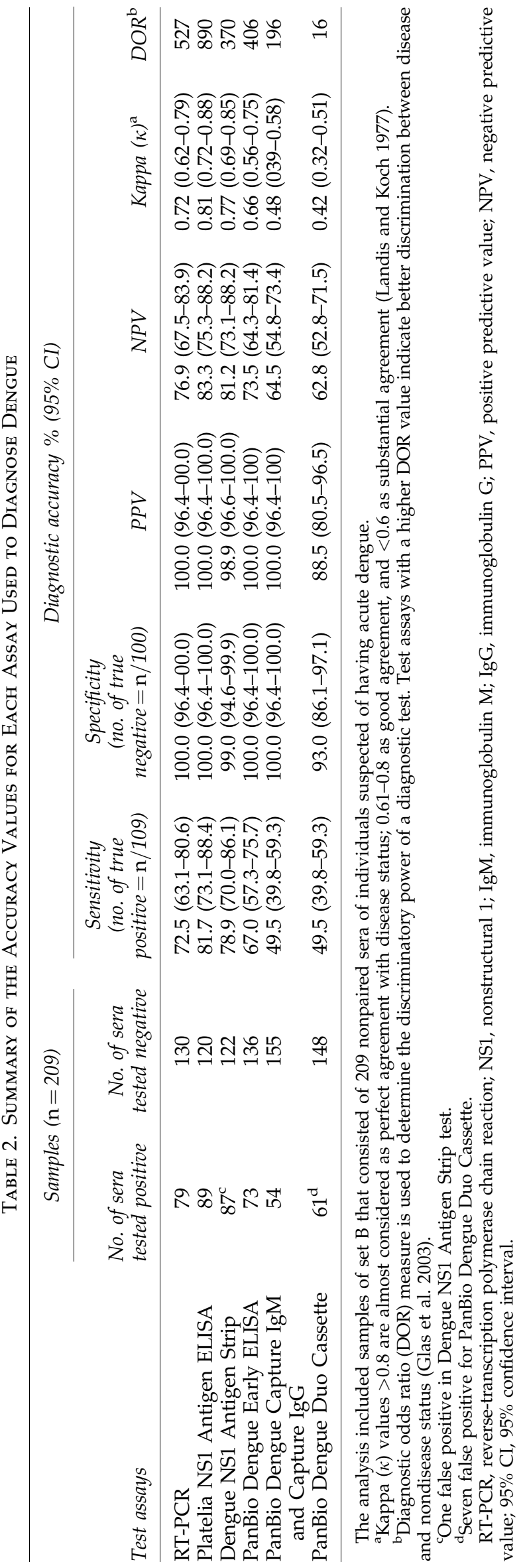

patients with dengue RT-PCR-negative acute sera $(n=60)$, none seroconverted during the convalescence.

Of 209 nonpaired samples of set B, 109 (52.1\%) were positive for DENV. They included 79 sera that were positive by RT-PCR (72.4\%) and 30 sera that were RT-PCR negative but positive for PanBio Capture IgM and/or Capture IgG assays. The remaining 100 sera were confirmed as negative for acute dengue infection based on RT-PCR and Capture IgM/IgG assays. Of 79 RT-PCR-positive sera, 77 were successfully serotyped, which showed all four serotypes: DENV1 $(n=32)$, DENV2 $(n=35), \operatorname{DENV3}(n=9)$, and DENV4 $(n=1)$. All samples were collected within a mean number of 3.49 days from the onset of fever (95\% CI: 0.95-6.03 days).

\section{Sensitivity of dengue diagnostic assays}

The performance of the dengue diagnostic assays was evaluated using paired samples (Set A). Of all assays tested, RT-PCR assay described by Lai et al. (2007) demonstrated $100 \%$ sensitivity (Fig. 1) and specificity in acute sera collected within the first 3 days of fever. Sensitivity of RT-PCR, however, declined over time: $45.8 \%$ in days $5-6$ and $8.3 \%$ in days 7-8 sera (Fig. 1).

In contrast, dengue IgM antibody detection assays (Capture IgM or Dengue Duo) showed only $17.3-21.2 \%$ of sensitivity among acute sera collected within the first 3 days of fever. These assays achieved high sensitivity (83.3-100\%) of diagnosing dengue in convalescent samples of RT-PCRpositive sera, obtained after 5 days of the onset of fever.

The dengue NS1 antigen assays evaluated in this study demonstrated $71.2-82.7 \%$ sensitivity of detecting dengue in acute sera collected within the first 3 days of fever. Their sensitivity remained persistently high (66.7-79.2\%) until the sixth day after the onset of fever (Fig. 1). In general, Platelia assay showed the highest sensitivity $(>75.0 \%)$ during the first 8 days of fever, followed by NS1 Strip $(>69.2 \%)$ and Dengue Early ( $>66.3 \%$ ) assays. A high concordance of results $(96.4 \%$, 216/224) was observed between the Platelia and NS1 Strip assays. Similarly, the concordance between the Platelia and Dengue Early assays was $93.75 \%(210 / 224)$.

Of 60 dengue-negative sera of sample set $\mathrm{A}$, none of the acute and convalescent sera showed positive results by any of the three dengue NS1 antigen assays evaluated, indicating $100 \%$ specificity for the detection of DENV.

\section{Performance of dengue NS1 antigen assays among routine diagnostic samples}

In this study, 109 dengue-positive and 100 denguenegative, nonpaired samples (set B) were used to ascertain the robustness of three NS1 assays in diagnosing dengue in primary healthcare settings in Singapore.

Overall, dengue NS1 detection assays showed the highest sensitivity of detecting DENV: Platelia assay (81.7\%), NS1 Strip assay $(78.9 \%)$, and Dengue Early assay $(67.0 \%)$ when compared with RT-PCR (72.5\%) and dengue IgM Capture and IgM/IgG Dengue Duo assays (49.5\%). There were 18 sera collected between fourth and seventh days after the onset of fever that were RT-PCR negative but positive for dengue by NS1 assays. Platelia assay detected DENV in all 18 sera, whereas NS1 Strip and Dengue Early assays detected 16 and 11 sera, respectively. All except 1 of the 18 NS1-positive samples were positive for dengue by Capture IgM and/or 
Capture IgG assays. On the other hand, there were eight sera collected within the first 3 days of fever that were positive for dengue by RT-PCR, but negative by NS1 assays.

Both the Platelia and Dengue Early assays demonstrated $100 \%$ specificity; however, one false positive was detected using NS1 Strip. In comparison, RT-PCR and Dengue Duo detection assays demonstrated $0 \%$ and $7 \%$ false-positive rates, respectively.

As shown in Table 2, Cohen's kappa $(\kappa)$ values of more than 0.7 were observed for RT-PCR $(\kappa=0.72)$, Platelia $(\kappa=0.81)$, and NS1 Strip $(\kappa=0.77)$ assays, indicating a strong agreement of obtained results with the dengue infection status of patient sera. Dengue Early achieved a kappa value of 0.66 , indicating a slightly less but good agreement. In contrast, dengue IgM/ IgG detection assays demonstrated low kappa values (less than 0.5 ), indicating relatively low agreement of their results with dengue infection status.

The DOR measures the odds of positivity in a disease relative to the odds of positivity in nondiseased case (Glas et al. 2003). The DOR values reflect a similar pattern of performance among the evaluated assays in this study as the kappa values. The Platelia assay showed the highest DOR (890) among the dengue diagnostic assays tested (Table 2): RT-PCR $(\mathrm{DOR}=527)$, Dengue Early $(\mathrm{DOR}=406)$, NS1 Strip (DOR = 370), Capture IgM/Capture IgG $(\mathrm{DOR}=196)$, and Dengue Duo $(\mathrm{DOR}=16)$

\section{Serotype sensitivity of dengue NS1 antigen assays}

Of 129 dengue-positive sera serotyped by RT-PCR (set $A=52$, set $B=77$ ), the Platelia assay showed greater than $86 \%$ sensitivity (Table 3) in detecting all four serotypes (DENV1: 86.3\%, DENV2: 88.1\%, DENV3: $88.2 \%$, DENV4: 100.0\%). The differences observed for each serotype were not statistically significant (chi-square test: $\chi^{2}=0.35, p=0.950$ ). Similarly, no significant difference was observed in the sensitivity levels of the NS1 Strip test for detecting different dengue serotypes $\left(\chi^{2}=1.10, p=0.776\right)$. However, Dengue Early assay showed a significant difference in the sensitivity of detection between DENV1 and DENV3 serotypes $\left(\chi^{2}=19.47, p<0.05\right)$.

\section{Performance of dengue NS1 assays in primary and secondary dengue infections}

Accordingly to the Recife classification, 46 (28.6\%) of 161 dengue-positive sera of sets A and B were classified as secondary dengue infections. As shown in Table 4, dengue NS1 antigen assays, in general, showed higher sensitivity of detecting primary $(75.7-93.0 \%)$ than secondary $(47.8-54.4 \%)$ dengue infections. The detection rates of all three dengue NS1 antigen assays were significantly higher (Platelia: $\chi^{2}=33.32$, $p<0.05$; NS1 Strip: $\chi^{2}=35.06, p<0.05 ;$ Dengue Early: $\left.\chi^{2}=9.90, p<0.05\right)$ in patients with primary than secondary dengue infections.

\section{Discussion}

Primary healthcare settings offer an excellent opportunity for the early diagnosis of dengue infections which helps in better patient management. Early diagnosis also enhances disease surveillance and control by accurate and prompt identification of active disease clusters. This is important in Singapore where dengue is endemic and is more commonly 
Table 4. Sensitivity of Three Dengue Nonstructural 1 Antigen Detection Assays According to Dengue Infection Status (Primary Dengue Infection Versus Secondary Dengue Infection)

\begin{tabular}{|c|c|c|c|c|}
\hline \multirow[b]{2}{*}{ Dengue infection status } & \multicolumn{2}{|c|}{ Primary dengue $^{\mathrm{a}}(\mathrm{n}=115)$} & \multicolumn{2}{|c|}{ Secondary dengue ${ }^{\mathrm{b}}(\mathrm{n}=46)$} \\
\hline & $\begin{array}{c}\text { No. of } \\
\text { positive tests }\end{array}$ & $\begin{array}{l}\text { Sensitivity \% } \\
\quad(95 \% \mathrm{CI})\end{array}$ & $\begin{array}{c}\text { No. of } \\
\text { positive tests }\end{array}$ & $\begin{array}{l}\text { Sensitivity \% } \\
\quad(95 \% \mathrm{CI})\end{array}$ \\
\hline Bio-Rad Platelia Dengue NS1 Antigen ELISA & 107 & $93.0(86.8-97.0)$ & 25 & $54.4(39.0-69.1)$ \\
\hline Bio-Rad Dengue NS1 Antigen Strip & 104 & $90.4(83.5-95.1)$ & 22 & $47.8(32.9-63.1)$ \\
\hline PanBio Dengue Early ELISA & 87 & $75.7(66.8-83.2)$ & 23 & $50.0(34.9-65.1)$ \\
\hline
\end{tabular}

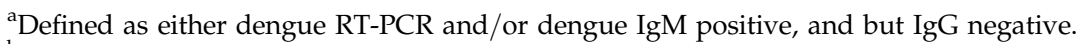

${ }^{\mathrm{b}}$ Defined as having high levels (PanBio units $>22$ ) of dengue IgG antibody (hemagglutination inhibition titer of $>2560$ ) for sample collected within the first 8 days of fever.

reported among adults, who are more prone to develop severe complications, especially when associated with comorbid conditions such as diabetes and hypertension (Lee et al. 2006).

Currently, there is no single diagnostic assay that can accurately detect dengue throughout the acute and convalescence phases. The performance of all available methods varies depending on the duration of clinical disease. It is thus important to determine the performance of individual tools within the course of illness, as the right tools should be deployed at the right time to ensure the maximal diagnostic capability among individuals. This is the first report on the evaluation of all three commercial NS1 kits together with other commonly used diagnostic tools at the primary healthcare setting. Our study showed that NS1 detection was the most sensitive test at the primary healthcare setting. Many public health laboratories in Singapore, including EHI, have long relied on RT-PCR technology for the diagnosis and serotyping of DENV infections. Though highly specific and sensitive, especially during the early phase of the clinical disease, RT-PCR requires skilled personnel, sophisticated equipments, and laboratory facilities. As the majority of primary healthcare settings in Singapore lack the opportunity to perform RT-PCR, the routine diagnosis of dengue fever mainly relies upon the rapid diagnostic test assays such as PanBio Dengue Duo assay.

Our results showed that the sensitivity of RT-PCR tend to decline after 3 days of the onset of fever, indicating that PCR technology would be effective for dengue diagnosis only during the very early stages of the disease. Moreover, dengue IgM/IgG detection assays also showed low sensitivity $(<32.0 \%)$ for sera collected during the first 5 days of fever. However, NS1 antigen detection assays showed consistently high sensitivity of DENV detection among sera collected within the first 6 days of fever. Based on these observations, dengue NS1 antigen assays appeared to be an alternative to RT-PCR and antibody testing in the routine diagnosis of dengue in Singapore.

To further evaluate the suitability of dengue NS1 assays in routine diagnosis of dengue fever in Singapore, we calculated the sensitivity and DOR among 209 nonpaired, denguesuspected sera collected within 8 days of the onset of fever (Table 2). DOR measures the odds of positivity in a disease status relative to the odds of positivity in a nondiseased status and thereby reflects the discriminatory power of diagnostic test assays. DOR values range from 0 to infinity, with higher values indicating better discriminatory test performance (Glas et al. 2003). According to our analysis, the Platelia assay achieved the highest sensitivity $(81.7 \%)$, followed by NS1 Strip (78.9\%) and Dengue Early assays (67.0\%). The sensitivity of both the Platelia and NS1 Strip assays was even higher than that of RT-PCR $(72.5 \%)$ among the test sera. This is mainly because approximately $95 \%$ of patients suspected of having dengue, in Singapore, seeks treatment from the general practitioners around 3.49 days (95\% CI: 0.95-6.03) after the onset of fever. Similar to sensitivity data, NS1 assays demonstrated high discriminatory power. On the other hand, antibody detection assays, though very commonly used worldwide, achieved a sensitivity of only $50 \%$ among the samples. Serological dengue testing also faced some problems with a small percentage of secondary dengue-infected samples with low or no detectable dengue IgM (Kuno et al. 1991, Ruechusatsawat et al. 1994). Thus, a surveillance system that relies on IgM detection is not likely to reflect the transmission dynamics of the disease.

Further, performance of the Platelia and NS1 Strip assays demonstrated no significant difference in the sensitivity of detecting DENV-1, DENV-2, and DENV-3. Dussart et al. (2006) reported a similar finding for the performance of the Platelia Dengue NS1 Antigen ELISA assay. Though our analysis showed reduced sensitivity of Dengue Early assay for the detection of DENV3 serotype, more samples are needed to confirm this observation.

Our observation of a reduced sensitivity of NS1 assays for secondary dengue infections is in agreement with previous findings (Koraka et al. 2003, Kumarsamy et al. 2007, Blacksell et al. 2008). The possibility of anti-NS1 antibodies resulting from a previous infection in quenching the NS1 antigen of a current infection has been discussed to explain this observation (Valdes et al. 2000, Libraty et al. 2002, Koraka et al. 2003). Nevertheless, NS1 assay was found to have around 50\% sensitivity among secondary dengue infections in Singapore, where approximately $45 \%$ of dengue cases are secondary infections (Low et al. 2006). A factor that may influence the performance of NS1 assays would be the composition of Primary and Secondary dengue infections and health-seeking behavior of patients. Studies from various populations are needed for a comprehensive understanding of the performances of NS1 assays.

In comparison with the ELISA-based formats of NS1 assay, NS1 Strip assay has more favorable design characteristics such as ease of use, possibility of testing individual sample, rapidity, and minimal requirement of laboratory equipments. It also performed comparably to the ELISA-based NS1 assays. When comparing rapid tests, NS1 Strip assay was 23 times 
more discriminatory than PanBio Dengue Duo rapid diagnostic test in determining DENV infection in test sera.

Based on these findings, medical practitioners in Singapore are encouraged to request assays for dengue NS1 for patients within 5 days of fever onset and IgM assays for those beyond the fifth day after fever onset. This strategy would achieve an improved sensitivity of $87.2 \%$ in detection of dengue infection (sensitivity of dengue NS1 assay: $86.5 \%$; sensitivity of IgM: $90.0 \%)$. However, RT-PCR continues to play a significant role in serotyping and virological surveillance of DENV ( $\mathrm{Ng}$ et al. 2007). Our study demonstrates the advantage and importance of a comprehensive, local evaluation before applying any commercial diagnostic test in a clinical setting.

\section{Acknowledgments}

The authors thank the National Environmental Agency and Singapore Polytechnic for funding the project through research grants; the Inverness Medical Innovations and Bio-Rad Laboratories (Singapore) Pvt. Ltd. for providing the Dengue NS1 Kits for evaluation; the Early Dengue Team and the EHI Disease Surveillance Team, who has put in concerted effort in obtaining and processing the clinical samples; and Dr. Chanditha Hapuarachchi, for patiently revising the article and contributing many helpful suggestions.

\section{Disclosure Statement}

No competing financial interests exist.

\section{References}

Alcon, S, Talarmin, A, Debruyne, M, Falconar, A, et al. Enzymelinked immunosorbent assay specific to dengue virus type 1 nonstructural protein NS1 reveals circulation of the antigen in the blood during the acute phase of disease in patients experiencing primary or secondary infections. J Clin Microbiol 2002; 40:376-381.

Blacksell, SD, Mammen, MP, Jr., Thongpaseuth, S, Gibbons, R, et al. Evaluation of the PanBio dengue virus nonstructural 1 antigen detection and immunoglobulin $\mathrm{M}$ antibody enzymelinked immunosorbent assays for the diagnosis of acute dengue infections in Laos. Diagn Microbiol Infect Dis 2008; 60:43-49.

Calisher, CH, Karabatsos, N, Dalrymple, JM, Shope, RE, et al. Antigenic relationships between flavivirus a determined by cross-neutralization tests with polyclonal antisera. J Gen Virol 1989; 70:37-43.

Cordeiro, MT, Braga-Neto, U, Nogueira, RMR, Marques, ETA, Jr. Reliable classifier to differentiate primary and secondary acute dengue infection based on IgG ELISA. PLoS ONE 2009; 4:e4945.

Dussart, P, Labeau, B, Lagathu, G, Louis, P, et al. Evaluation of an enzyme immunoassay for detection of dengue virus NS1 antigen in human serum. Clin Vaccine Immunol 2006; 13:1185-1189.

Glas, AS, Lijmer, JG, Prins, MH, Bonsel, GJ, et al. The diagnostic odds ratio: a single indicator of test performance. J Clin Epidemiol 2003; 56:1129-1135.

Gubler, DJ. The global emergence/resurgence of arboviral diseases as public health problems. Arch Med Res 2002; 33:330342.

Guzman, MG, Kouri, G. Advances in dengue diagnosis. Clin Diagn Immunol 1996; 3:621-627.
Halstead, SB. Antibody, macrophages, dengue virus infection, shock, and hemorrhagic: a pathogenic cascade. Rev Infect Dis 1989; 11:830-839.

Huang, JL, Huang, JH, Shyu, RH, Teng, CW. High level expression of recombinant dengue viral NS-1 protein an its potential use as a diagnostic antigen. J Med Virol 2001; 65:553-560.

Koraka, P, Burghoorn-Maas, CP, Falconar, A, Setiati, TE, et al. Detection of immune-complex-dissociated nonstructural-1 antigen in patients with acute dengue virus infections. J Clin Microbiol 2003; 41:4154-4159.

Kumarasamy, V, Wahab, AH, Chua, SK, Hassan, Z, et al. Evaluation of a commercial dengue NS1 antigen ELISA for laboratory diagnosis of acute dengue virus infection. J Virol Methods 2007; 40:75-79.

Kuno, G, Gomez, I, Gubler, DJ. An ELISA procedure for the diagnosis of dengue infections. J Virol Methods 1991; 33:101113.

Lai, YL, Chung, YK, Tan, HC, Yap, HF, et al. Cost-effective realtime reverse transcriptase (RT-PCR) to screen for dengue virus followed by rapid single-tube multiplex RT-PCR for serotyping of virus. J Clin Microbiol 2007; 45:935-941.

Lanciotti, RS, Calisher, CH, Gubler, DJ, Chang, GJ, et al. Rapid detection and typing of dengue viruses from clinical samples by using reverse transcriptase-polymerase chain reaction. J Clin Microbiol 1992; 30:545-551.

Landis, JR, Koch, GG. The measurement of observer agreement for categorical data. Biometrics 1977; 33:159-174.

Lapphra, K, Sangcharaswichai, A, Chopkephaibukit, K, Tiengrim, S, et al. Evaluation of an NS1 antigen detection for diagnosis of acute dengue infection in patients with acute febrile illness. Diagn Microbiol Infect Dis 2008; 4:387-391.

Lee, MS, Hwang, KP, Chen, TC, Lu, PL, et al. Clinical characteristics of dengue and dengue haemorrhagic fever in a medical center of southern Taiwan during the 2002 epidemic. J Microbiol Immunol Infect 2006; 39:121-129.

Libraty, DH, Young, PR, Pickering, D, Endy, TP, et al. High circulating levels of the dengue virus nonstructural protein NS1 early in dengue illness correlate with the development of dengue hemorrhagic fever. J Infect Dis 2002; 186:1165-1168.

Low, JG, Ooi, EE, Tolfvenstam, T, Leo, YS, et al. Early dengue infection and outcome study (EDEN)—study design and preliminary findings. Ann Acad Med Singapore 2006; 35:783-789.

Monath, TP, Heonz, FX. Flavivirus. In: Fields, BN, Knipe, BM, Howley, PM, eds. Fields' Virology, 3rd edition. Philadelphia: Lippincott-Raven, 1996:961-1034.

Ng, LC, Lai, YC, Cheng, C, Barkham, T. Spatial and temporal distribution of dengue serotypes in Singapore. Epidemiol News Bull 2007; 33:1-3.

Pan American Health Organization. Dengue and Dengue Hemorrhagic Fever in the Amercas: Guidelines for Prevention and Control. Scientific Publication 548. Washington, DC: Pan American Health Organization, 1994.

Ruechusatsawat, S, Morita, K, Tanaka, M, Vougcheree, S, et al. Daily observation of antibody levels among dengue patients detected by enzyme-linked immunosorbent assay (ELISA). Jpn Trop Med Hyg 1994; 22:9-12.

Valdes, K, Mayling, A, Pupo, M, Susana, V, et al. Human dengue antibodies against structural and non-structural proteins. Clin Diagn Lab Immunol 2000; 7:856-857.

Vorndam, V, Kuno, G. Laboratory diagnosis of dengue virus infections. In: Gubler, DJ, Kuno, G, eds. Dengue and Dengue Hemorrhagic Fever. London: CAB International, 1997:313-334.

Winkler, G, Maxwell, SE, Ruemmler, C, Stollar, V. Newly synthesized dengue-2 virus nonstructural protein NS1 is a soluble 
protein but becomes partially hydrophobic and membraneassociated after dimerization. Virology 1989; 171:302-305.

World Health Organization. Dengue and dengue haemorrhagic fever; fact sheet no. 117, revised March 2009. Geneva: World Health Organization, 2009. Available at www.who.int/ mediacentre/factsheets/fs117/en/, accessed August 31, 2009.

Xu, H, Di, B, Pan, YX, Qiu, LW, et al. Serotype 1-specific monoclonal antibody-based antigen capture immunoassay for detection of circulating nonstructural protein NS1: Implications for early diagnosis and serotyping of dengue virus infections. J Clin Microbiol 2006; 44:2872-2878.

Young, PR, Hilditch, PA, Bletchly, C, Halloran, W. An antigen capture enzyme-linked immunosorbent assay reveals high levels of the dengue virus protein NS1 in the sera of infected patients. J Clin Microbiol 2000; 38:1053-1057.

Address correspondence to: Lee-Ching $\mathrm{Ng}$

National Environment Agency Environmental Health Institute

11 Biopolis Way \#06-05/08 Helios Block Singapore 138667 Singapore

E-mail: ng_lee_ching@nea.gov.sg 